\title{
A Case Report of Cardio-Respiratory Arrest Suspected of Amniotic Fluid Embolism (Anaphylactoid Syndrome of Pregnancy)
}

\author{
ARTICLE INFO \\ Article Type \\ Case Report \\ Authors \\ Mojtaba Mir Aboutalebi ${ }^{1}$, MD * \\ Mohammad Amin Samet ${ }^{1}$, MD \\ Javad Amini Mahabadi 1,3, iD PhD \\ Mohammad Reza Nateghi ${ }^{2}$ (ID MD \\ Sarem Fertility \& Infertility \\ Research Center (SAFIR), Sarem \\ Women's Hospital, Iran University \\ of Medical Sciences (IUMS), \\ Tehran, Iran. \\ 2 Sarem Fertility \& Infertility \\ Research Center (SAFIR) \& Sarem \\ Cell Research Center (SCRC), \\ Sarem Women's Hospital, Iran \\ University of Medical Sciences \\ (IUMS), Tehran, Iran. \\ $3 \mathrm{PhD}$ of reproductive biology, \\ Gametogenesis Research Center, \\ Kashan University of Medical \\ Sciences, Kashan, Iran.
}

\section{ABSTRACT}

Introduction: Amniotic fluid embolism (Anaphylactoid Syndrome of Pregnancy) is a rare and unpredictable complication of pregnancy that occurs during or postparturition. This complication occurs when amniotic fluid enters mother blood, which this condition is rare and its main symptom is cardiopulmonary arrest. Because maternal mortality associated with this complication is so high, early detection and prompt treatment are important for the improvement of prognosis.

Case presentation: A 37-year-old pregnant woman, gravida 2 para 2, experienced cardiac and respiratory arrest at the end of the cesarean operation and 45 minutes after spinal anesthesia and then baby exhaust. The baby was healthy. Mother had a height of $170 \mathrm{~cm}$, weight $117 \mathrm{~kg}$, and BMI of more than 40 that CPR was started promptly and after 10 minutes of necessary evaluations, the patient had vital signs and sinus rhythm. The patient was discharged in good general condition.

Conclusion: Initial supportive care is important if amniotic fluid embolism (AFE) is suspected. Early diagnosis and aggressive management of the patient with cardiopulmonary resuscitation will be associated with increased survival and reduced morbidity and mortality.

Keywords: Amniotic Fluid Embolism (AFE); Anaphylactoid Syndrome of Pregnancy (ASP); Cardiopulmonary Arrest; Cesarean; Case Report.
*Corresponding Author

Address: Sarem Women Hospital, Basij Square, Phase 3 , EkbatanTown, Tehran, Iran. Postal code: 1396956111

Phone: +98 (21) 44670888

Fax: +98 (21) 44670432

\section{Article History}

Received: December 06, 2020

Accepted: December 15, 2020

e Published: August 08, 2021

Copyright(C) 2021, ASP Ins. This open-access article is published under the terms of the Creative Commons Attribution-Noncommercial 4.0 International License which permits Share (copy and distribute the material in any medium or format) and Adapt (remix, transform, and build 
تهاجمى بيمار با احيا قلبى-ريوى، با افزايش بقا و كاهش عوارض و مركَ و

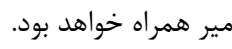

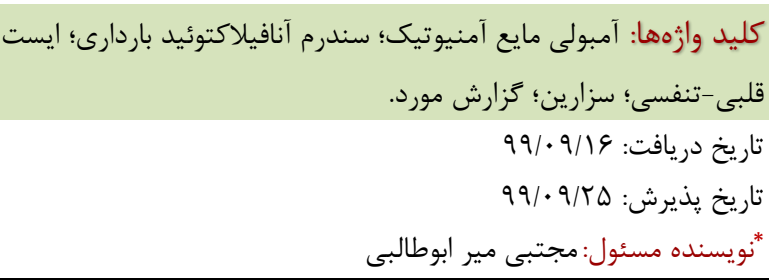

مقدمه

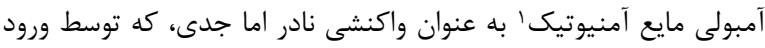

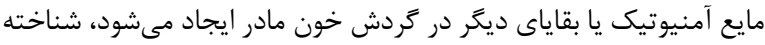

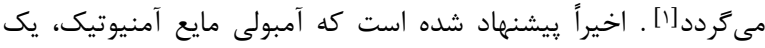

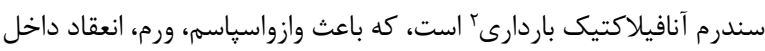

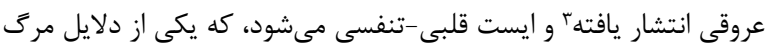

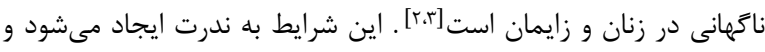

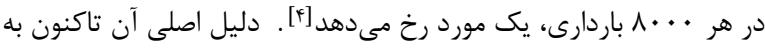

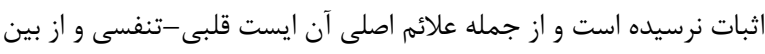

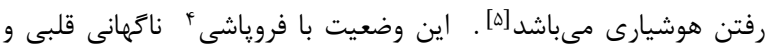

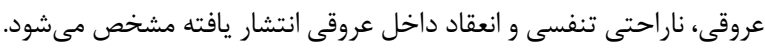

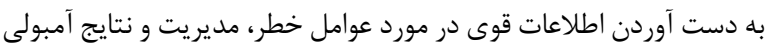

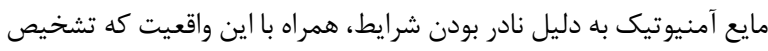

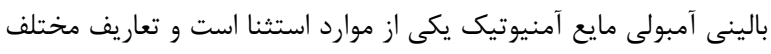

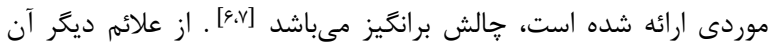

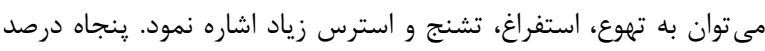

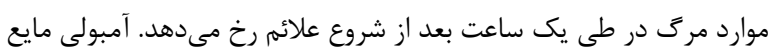

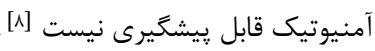

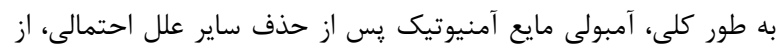

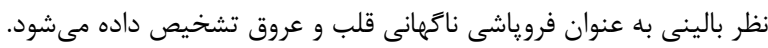

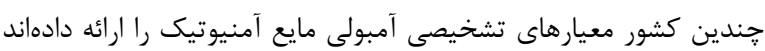

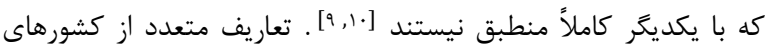

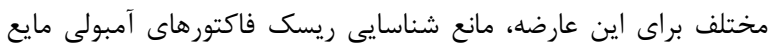

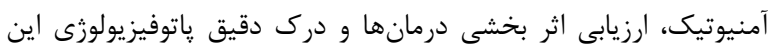

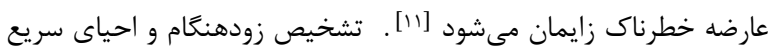

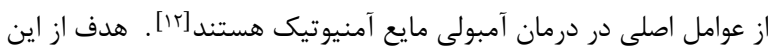

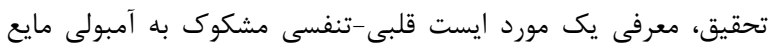

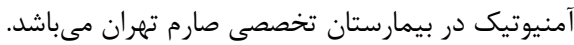

Disseminated Intravascular Coagulation (DIC) ${ }^{r}$ Collapse
كزارش يك مورد ايست ق قلبى تنفسى مشكوى به آمبولى مايع آنافيلاكتوئيد آمنيو تيك سندرم

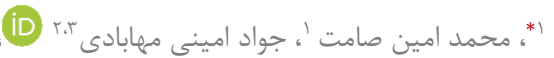
(iD)

محمد رضا ناطقى مجتي ميرالبي مركز تحقيقات بارورى و نابارورى صارم، بيمارستان فوق تخصصى صارم، دانشخاه علوم يزشكى ايران، تهران، ايران.

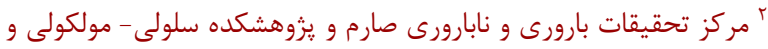

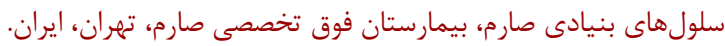

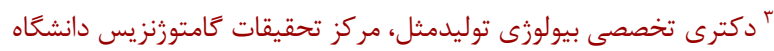
علوم يزشكى كاشان، كاشان، ايران.

جكيده مقدمه: آمبولى مايع آمنيوتيك (سندرم آنافيلاكتوئيد باردارى) يك عارضه

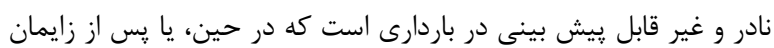

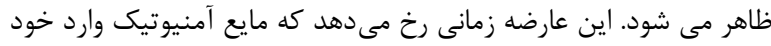

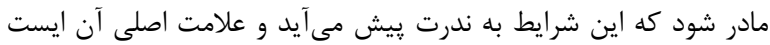

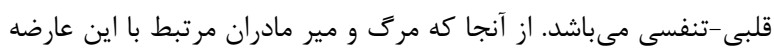

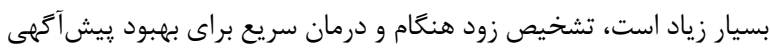

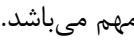

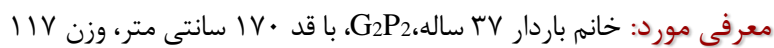

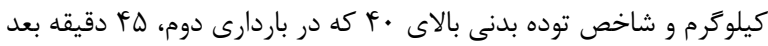

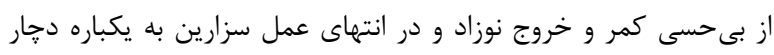
ايست قلبى و تنفسى شد، كه با تشخيص اوليه آمبولى مايع آمنيوتيك،

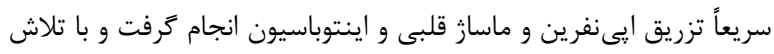

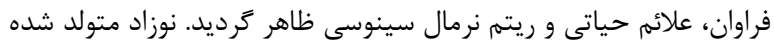

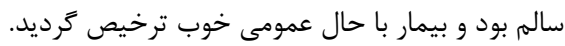

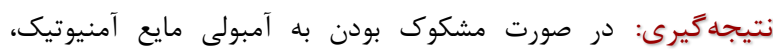
مراقبتهاى حمايتى اوليه مهمم است. تشخيص زودهنگام و مديريت 


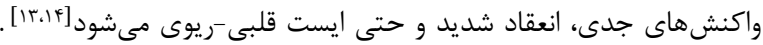

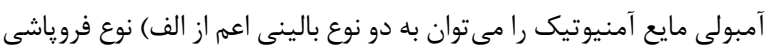

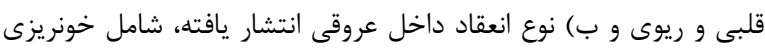

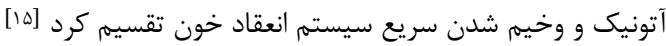

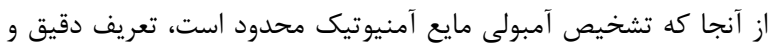

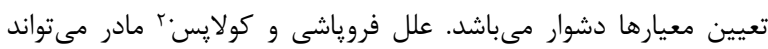

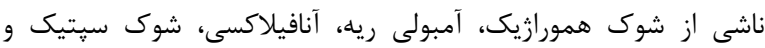

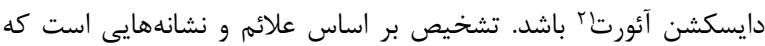

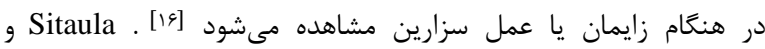

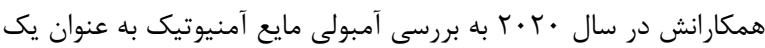

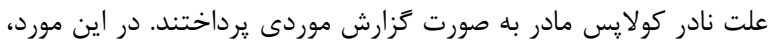

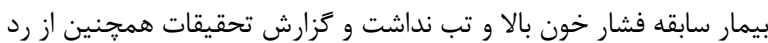

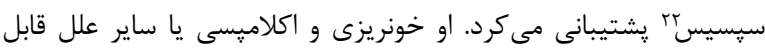

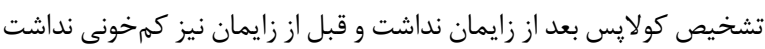

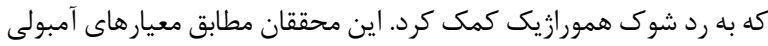

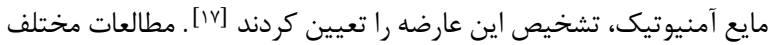

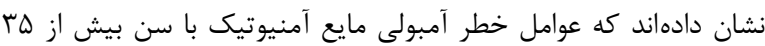
سال، جندقلويى، سزارين، خونريزى قبل از زايمان، اكلاميسى، إنى القا زايمان،

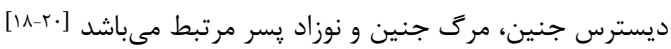

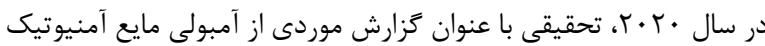

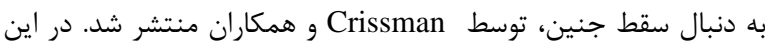

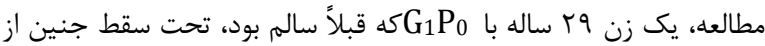

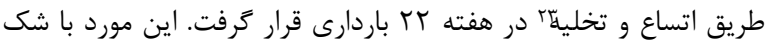

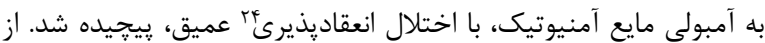

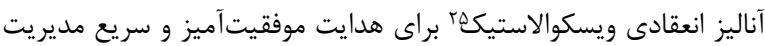

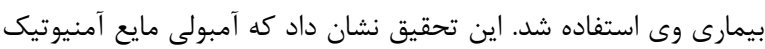

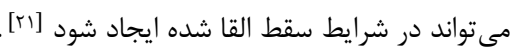

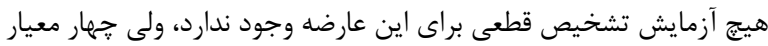

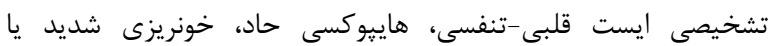
اختلالات انعقادى، در طول زايمان يا سزارين بدون توضيح خاص دئيكر،

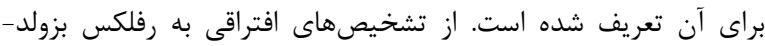

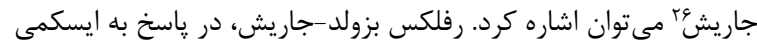

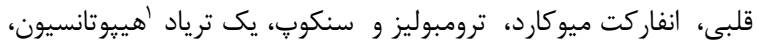
براديكاردى و گشاد شدن عروق كرونر ايجاد مئمايد. نكته ديكر اينكه در

Lasix (Furosemide) Clear

Anaphylactoid Syndrome of Pregnancy (ASP)

Complement System ${ }^{19}$ Collapse ${ }^{r}$.

Aortic Dissection ${ }^{r}$ Sepsis $^{r r}$

Dilation and Curettage (D\&C) ${ }^{r}$

Coagulopathy ${ }^{r s}$

Viscoelastic Point-of-Care (POC) Analysis ${ }^{\text {ro }}$ Bezold-Jarish Reflex (BJR)
كزارش مورد

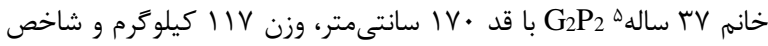

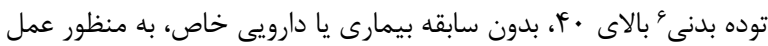

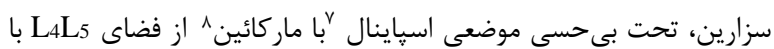

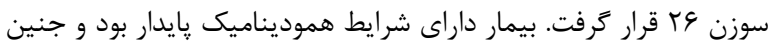

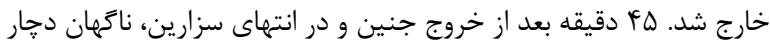

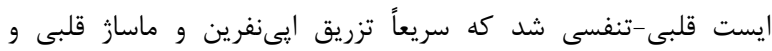

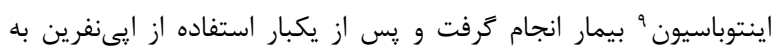

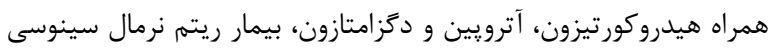

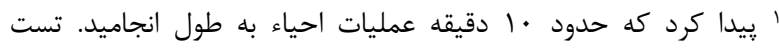
ABG "

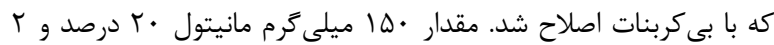

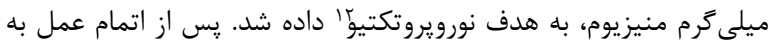

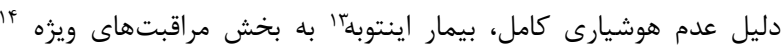

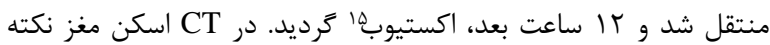

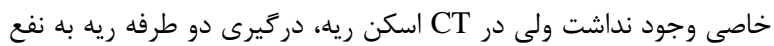

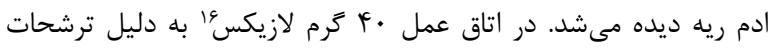

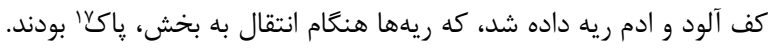

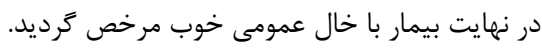

بحث

يكى از عوارض نادر حين باردارى، آمبولى مايع آمنيوتيك است كه متاسفانه

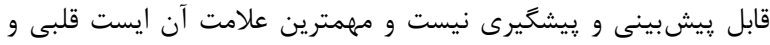

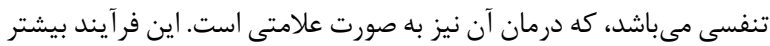

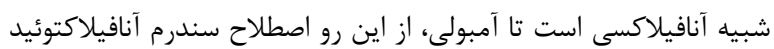

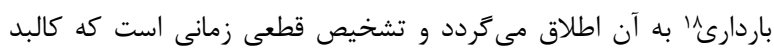

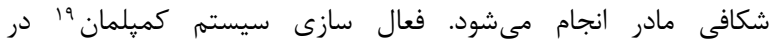

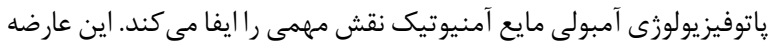

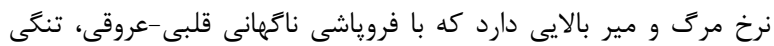

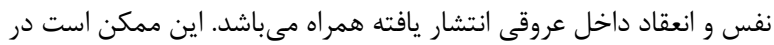

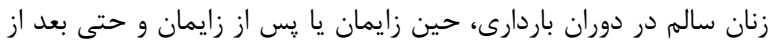

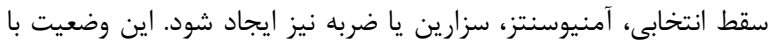

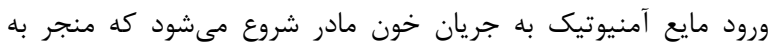

Gravidity and Parity Body Mass Index (BMI)

Spinal Anesthesia

Marcaine (Bupivacaine) Intubation Sinus Rhythm' Arterial Blood Gas (ABG) ' Neuroprotective' Intubate' Intensive Care Unit (ICU) Extubate 
1. Stafford I, Sheffield J. Amniotic fluid embolism Obstet Gynecol Clin North Am. 2007;34(3):54553.

2. Kanayama N, Inori J, Ishibashi-Ueda H, Takeuchi M, Nakayama M, Kimura S, et al. Maternal death analysis from the Japanese autopsy registry for recent 16 years: significance of amniotic fluid embolism. J Obstet Gynaecol Res. 2011;37(1):5863.

3. Busardò FP, Frati P, Zaami S, Fineschi V. Amniotic fluid embolism pathophysiology suggests the new diagnostic armamentarium: $\beta$-tryptase and complement fractions C3-C4 are the indispensable working tools. Int J Mol Sci. 2015;16(3):6557-70.

4. Kaur K, Bhardwaj M, Kumar P, Singhal S, Singh T, Hooda S. Amniotic fluid embolism. J Anaesthesiol Clin Pharmacol. 2016;32(2):153.

5. Sullivan E, Vaughan G, Knight M. AUSTRALASIAN MATERNITY OUTCOMES SURVEILLANCE SYSTEM (AMOSS) 2010-2011: A013. J Paediatr Child Health. 2012;48.

6. Fitzpatrick KE, Tuffnell D, Kurinczuk JJ, Knight M Incidence, risk factors, management and outcomes of amniotic-fluid embolism: a population-based cohort and nested casecontrol study. BJOG An Int J Obstet Gynaecol. 2016;123(1):100-9.

7. Schaap T, Bloemenkamp K, Deneux-Tharaux C, Knight M, Langhoff-Roos J, Sullivan E, et al. Defining definitions: a Delphi study to develop a core outcome set for conditions of severe maternal morbidity. BJOG An Int J Obstet Gynaecol. 2019;126(3):394-401.

8. Rudra A, Chatterjee S, Sengupta S, Nandi B, Mitra J. Amniotic fluid embolism. Indian J Crit care Med peer-reviewed, Off Publ Indian Soc Crit Care Med. 2009;13(3):129.

9. McDonnell N, Knight M, Peek MJ, Ellwood D, Homer CSE, McLintock C, et al. Amniotic fluid embolism: an Australian-New Zealand population-based study. BMC Pregnancy Childbirth. 2015;15(1):1-7.

10. Stafford IA, Moaddab A, Dildy GA, Klassen M, Berra A, Watters C, et al. Amniotic fluid embolism syndrome: analysis of the Unites States International Registry. Am J Obstet Gynecol MFM. 2020;2(2):100083.

11. Clark SL, Romero R, Dildy GA, Callaghan WM, Smiley RM, Bracey AW, et al. Proposed diagnostic criteria for the case definition of amniotic fluid embolism in research studies. Am J Obstet Gynecol. 2016;215(4):408-12.

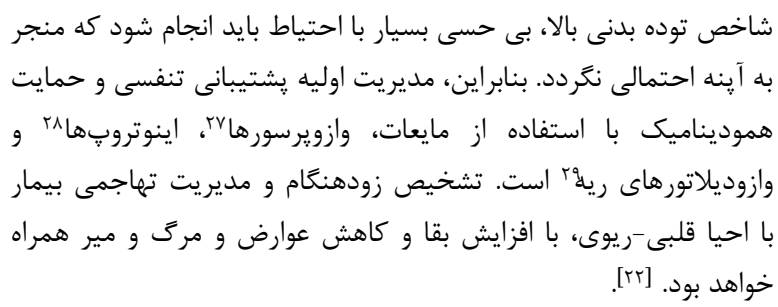


12. Kinishi Y, Ootaki C, Iritakenishi T, Fujino Y. A case of amniotic fluid embolism successfully treated by multidisciplinary treatment. JA Clin reports. 2019;5(1):1-4.

13. Lee JH, Yang HJ, Kim J-H, Lee S-Y, Gill HJ, Kim B-K, et al. Amniotic fluid embolism that took place during an emergent cesarean section-A case report. Korean J Anesthesiol. 2010;59(Suppl):S158.

14. Gilmore DA, Wakim J, Secrest J, Rawson R Anaphylactoid syndrome of pregnancy: a review of the literature with latest management and outcome data. AANA J. 2003;71(2).

15. Kanayama N, Tamura N. Amniotic fluid embolism: pathophysiology and new strategies for management. J Obstet Gynaecol Res. 2014;40(6):1507-17.

16. Benson MD. Current concepts of immunology and diagnosis in amniotic fluid embolism. Clin Dev Immunol. 2012;2012.

17. Sitaula S, Das D, Sitaula S, Chhetry M. Amniotic fluid embolism: A rare cause of maternal collapse-A case report. Clin Case Reports. 2020;8(12):3359.

18. Abenhaim HA, Azoulay L, Kramer MS, Leduc L. Incidence and risk factors of amniotic fluid embolisms: a population-based study on 3 million births in the United States. Am J Obstet Gynecol. 2008;199(1):49-e1.

19. Fitzpatrick KE, Van Den Akker T, Bloemenkamp KWM, Deneux-Tharaux C, Kristufkova A, Li Z, et al. Risk factors, management, and outcomes of amniotic fluid embolism: A multicountry, population-based cohort and nested case-control study. PLoS Med. 2019;16(11):e1002962.

20. Toy H. Amniotic Fluid Embolism. Eur J Gen Med. 2009;6(2).

21. Crissman HP, Loder C, Pancaro C, Bell J. Case report of amniotic fluid embolism coagulopathy following abortion; use of viscoelastic point-ofcare analysis. BMC Pregnancy Childbirth. 2020;20(1):1-7.

22. Pacheco LD, Saade G, Hankins GD V, Clark SL, (SMFM S for M-FM. Amniotic fluid embolism: diagnosis and management. Am J Obstet Gynecol. 2016;215(2):B16-24. 